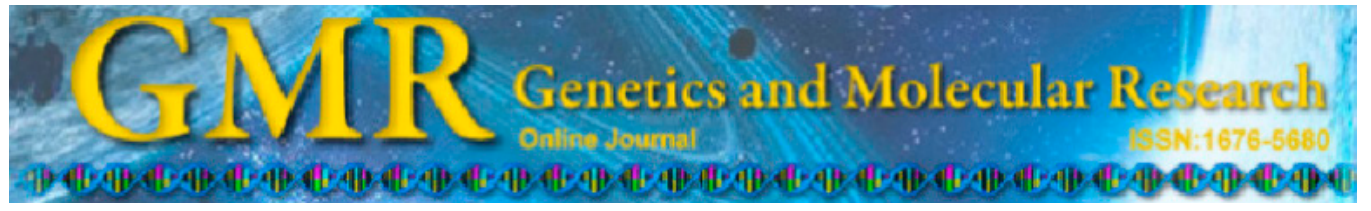

\title{
Evaluation of three different promoters driving gene expression in developing chicken embryo by using in vivo electroporation
}

\author{
C.Q. Yang ${ }^{1}$, X.Y. $\mathrm{Li}^{2}$, Q. $\mathrm{Li}^{3}$, S.L. Fu ${ }^{2}$, H. $\mathrm{Li}^{2}$, Z.K. Guo ${ }^{3}$, J.T. $\mathrm{Lin}^{2,3}$ and \\ S.T. Zhao ${ }^{1}$ \\ ${ }^{1}$ College of Veterinary Medicine, Northwest A\&F University, Yangling, China \\ ${ }^{2}$ College of Life Science and Technology, Xinxiang Medical University, \\ Xinxiang, China \\ ${ }^{3}$ Key Lab of Henan Province for Medical Tissue Regeneration, Xinxiang, China \\ Corresponding author: S.T. Zhao \\ E-mail: shantingzhao@126.com
}

Genet. Mol. Res. 13 (1): 1270-1277 (2014)

Received August 16, 2013

Accepted December 10, 2013

Published February 27, 2014

DOI http://dx.doi.org/10.4238/2014.February.27.12

\begin{abstract}
To investigate the variance of exogenous gene expression driven by different promoters by in vivo electroporation, 3 plasmid vectors carrying different promoters were selected, and their driving strength was compared in developing chicken embryos. The 3 promoters included: 1) the CAG promoter (containing the cytomegalovirus (CMV) immediate early enhancer and the chicken $\beta$-actin promoter), 2) the CMV promoter (the human CMV immediate early region enhancer), and 3) the SV40 promoter (Simian virus 40). The intensity of GFP expression driven by the 3 promoters was detected by fluorescence microscopy. The results clearly showed that the expression intensity of the reporter gene differed significantly among the 3 promoters. Chicken $\beta$-actin promoter induced the highest intensity of GFP expression, while SV40 promoter induced the lowest intensity. Our results indicate that plasmids with appropriate promoters should be carefully selected to obtain strong exogenous gene expression by in vivo electroporation.
\end{abstract}

Key words: Promoter; GFP; Chicken embryo; In vivo electroporation 


\section{INTRODUCTION}

The chicken embryo provides an excellent model system for studying gene function and regulation during embryonic development in vivo (Luo and Redies, 2005; Stern, 2005). Virus vectors, such as lentivirus (Hong et al., 2007) and adenovirus (Chang et al., 2013), have high transfection efficiency and long duration, both in vitro and in vivo; however, experiments with viruses are potentially dangerous to operators, and must be performed in special laboratories with strict regulations, which result in their limited application. Aside from virus vectors, plasmid vectors also provide a good source for gene expression. Compared to virus vectors, plasmid vectors are safer and easier for use in studies. Recently, the method of in vivo electroporation binding plasmid vector has become popular for studying the gain and loss of functions to exogenous gene expression, especially with respect to elucidating the development of the embryonic central nervous system (Muramatsu et al., 1997; Katahira and Nakamura, 2003; Chesnutt and Niswander, 2004; Nakamura et al., 2004; Das et al., 2006; Sauka-Spengler and Barembaum, 2008; Lin et al., 2011). In the same embryo after electroporation, the electroporated side represents the experimental area, while the other side serves as the control.

To study the gene function of the chicken embryo during brain or spinal cord development, it is better to transfect genes into the neural tube at incubation stage 18 to 24 of embryonic development. This is because the neural tube is a tight, high-resistance columnar epithelium with a closed lumen at this stage, which allows the spatial and temporal gene function of the developing nervous system to be investigated (Voiculescu et al., 2008). It has confirmed that electroporation significantly increases the efficiency of plasmid transfection. Because plasmids contain either reporter genes or target genes, in vivo electroporation is a popular method to assess gene gain of function (overexpression) or loss of function (RNA interference).

Promoters are a key element for controlling the level of gene expression of plasmids (Rhodius et al., 2012). In addition, the driving efficiency of promoters determines the expression strength of exogenous or reporter genes. Many plasmids with different promoters are currently available. Therefore, it is necessary to select the expression plasmid that has the appropriate promoter depending on whether strong, medium or weak gene expression is sought in a given experiment. In the present study, we evaluated the driving efficiency of 3 different promoters in developing chicken embryos. We aimed to identify the appropriate promoter inside plasmids that drives exogenous gene expression in the chicken embryonic system.

\section{MATERIAL AND METHODS}

\section{Embryo and tissue preparation}

Fertilized eggs were purchased from a local farm, and placed in an incubator (HWS150 , Jinghong, China) set to $37.5^{\circ} \mathrm{C}$ and $65 \%$ humidity. Embryos were staged according to Hamburger and Hamilton (1992), and studied at developmental stage 24 (E4), stage 27 (E5), stage 29 (E6), and stage 34 (E8). At least 3 embryos from each stage were studied for each experimental group. Embryos were removed from the eggs, and fixed in $4 \%$ formaldehyde solution on ice for 6-24 h, depending on the size of the embryos. After fixation, the tissues were immersed in $18 \%$ sucrose solution overnight. Then, specimens were embedded in Tissue-Tec O.C.T. compound (Sakura Finetek, USA), frozen in liquid nitrogen, and stored at $-80^{\circ} \mathrm{C}$. The samples were divided into sections of $20 \mu \mathrm{m}$ thickness on Poly-L-lysine-coated slides using a frozen slicer (Leica 1850, Germany). 


\section{Plasmid construction and extraction}

The plasmids pCAGGS-GFP (green fluorescent protein) and pEGFP were kept in our laboratory. The plasmid pCL-GFP was donated by Dr. Marcelle [Laboratoire de Génétique et de Physiologie du Développement (LGPD), Developmental Biology Institute of Marseille (IBDM), CNRS UMR 654, University Aix-Marseille II, Campus de Luminy, Case 907, 13288 Marseille Cedex 09, France. E-mail: marcelle@ibdm.univ-mrs.fr]. All 3 plasmids were extracted with an extraction kit (PurePlasmid Maxi kit, Lot:1412L) (Cwbio, Beijing, China).

\section{In vivo electroporation}

A specific in vivo electroporation protocol was followed. All steps were performed under a stereomicroscope. Fertilized eggs were incubated until stage 18 (E3). About 3-4 mL albumin was removed from each egg with a syringe, without disrupting the yolk. Then, the shell was carefully cut with curved scissors to create a 1-2-cm diameter window. The concentration of the plasmids was $0.5 \mu \mathrm{g} / \mu \mathrm{L}$ with $0.1 \%$ Fast Green dye (Sigma). Plasmid/Fast Green dye was injected and loaded into the neural tube lumen at the lumbar region with a mouth pipette until the dye filled the entire space. The electrodes were immediately placed parallel to the 2 lateral sides of the neural tube in the embryo. The false operation, without any plasmid, served as the negative control. A quantity of $0.25 \mu \mathrm{g} / \mu \mathrm{L}$ pCAGGS-GFP, combined with $0.5 \mu \mathrm{g} / \mu \mathrm{L}$ pEGFP or 0.5 $\mu \mathrm{g} / \mu \mathrm{L}$ pCL-GFP, was used to normalize the intensity of GFP expression. Electroporation was set at $18 \mathrm{~V}$ for $60 \mathrm{~ms}$, pausing for $100 \mathrm{~ms}$ between each pulse, with 6 pulses in total. Bubbles were observed near the electrodes if the experiment worked properly. After electroporation, the electrodes were carefully removed, and the egg was sealed well with tape. Then, the operated eggs were placed back into the incubator, and incubated until the desired stage was reached.

\section{Fluorescence imaging and data analysis}

The whole embryo was imaged under a stereo fluorescence microscope (LEICAM205FA, Germany) equipped with a digital camera (LEICA DFC425C, Germany). Sections were imaged under a fluorescence microscope (Nikon ECLIPSE 80i, Japan) equipped with a digital camera (LEICA DFC300FX, Germany). Digitized images were adjusted in contrast and brightness by the Photoshop software. GFP expression in the whole image was captured under the same conditions for all plasmids and the percentage area coverage was calculated using the Motic Images Advanced 3.2 Software. This value is used to indirectly infer the intensity of GFP expression.

\section{Statistical analysis}

Statistical significance was determined by the SPSS Statistics software using ANOVA. A P value of $<0.05$ was considered as significantly difference.

\section{RESULTS}

\section{Bioinformatic analysis of the 3 plasmid promoters}

A major difference was observed among the 3 plasmids (Figure 1). pCAGGS-GFP contains the CAG promoter (containing a cytomegalovirus (CMV) immediate early enhancer promoter and a chicken $\beta$-actin promoter) (Figure 1A). pEGFP contains the CMV promoter, 
which is the most commonly used for eukaryotic expression (Figure 1B). pCL-GFP promoter contains 2 promoters (the SV40 promoter driving reporter gene GFP expression, and chicken $\beta$-actin promoter driving target gene expression in the eukaryotic system) (Figure 1C). All the three plasmids carry the reporter gene GFP.
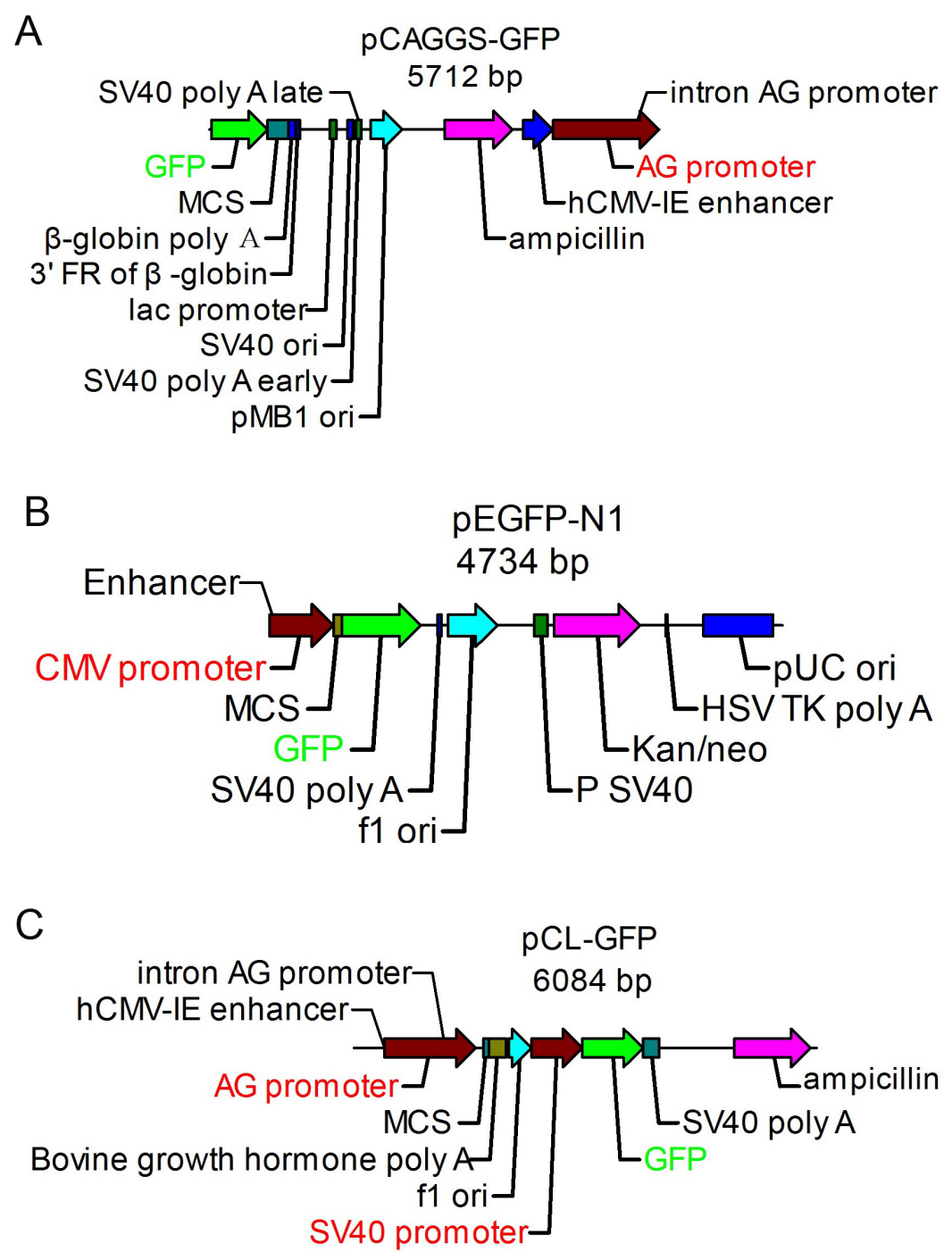

Figure 1. Vector maps of the three plasmids investigated. A. Map of pCAGGS-GFP plasmid. B. Map of pEGFP plasmid. C. Map of pCL-GFP plasmid.

\section{Differential expression of the 3 plasmids in developing chicken embryos}

The intensity of GFP expression for the same plasmid differed at stage 24 (E4; Figure 2A,D,G), stage 27 (E5; Figure 2B,E,H), and stage 29 (E6; Figure 2C,F,I). Furthermore, 
GFP expression also differed among the 3 plasmids at the same stages (Figure 2). At the same stage, pCAGGS-GFP had the strongest expression (Figure 2A,D,G), followed by pEGFP (Figure 2B,E,H), and finally pCL-GFP (Figure 2C,F,I). When using the same plasmid, the highest expression was observed during the first $48 \mathrm{~h}$ after electroporation (Figure 2D-F), with the expression gradually weakening after $72 \mathrm{~h}$ (Figure 2G-I). pCL-GFP expressed low GFP expression at stage 34 (E8), whereas GFP expression lasted longer after electroporation for pCAGGS-GFP and pEGFP. Fluorescence was still present in the pCAGGS-GFP plasmid until the chick hatched (data not shown). There was no significant difference in GFP expression among the 3 experimental control groups (data not shown).
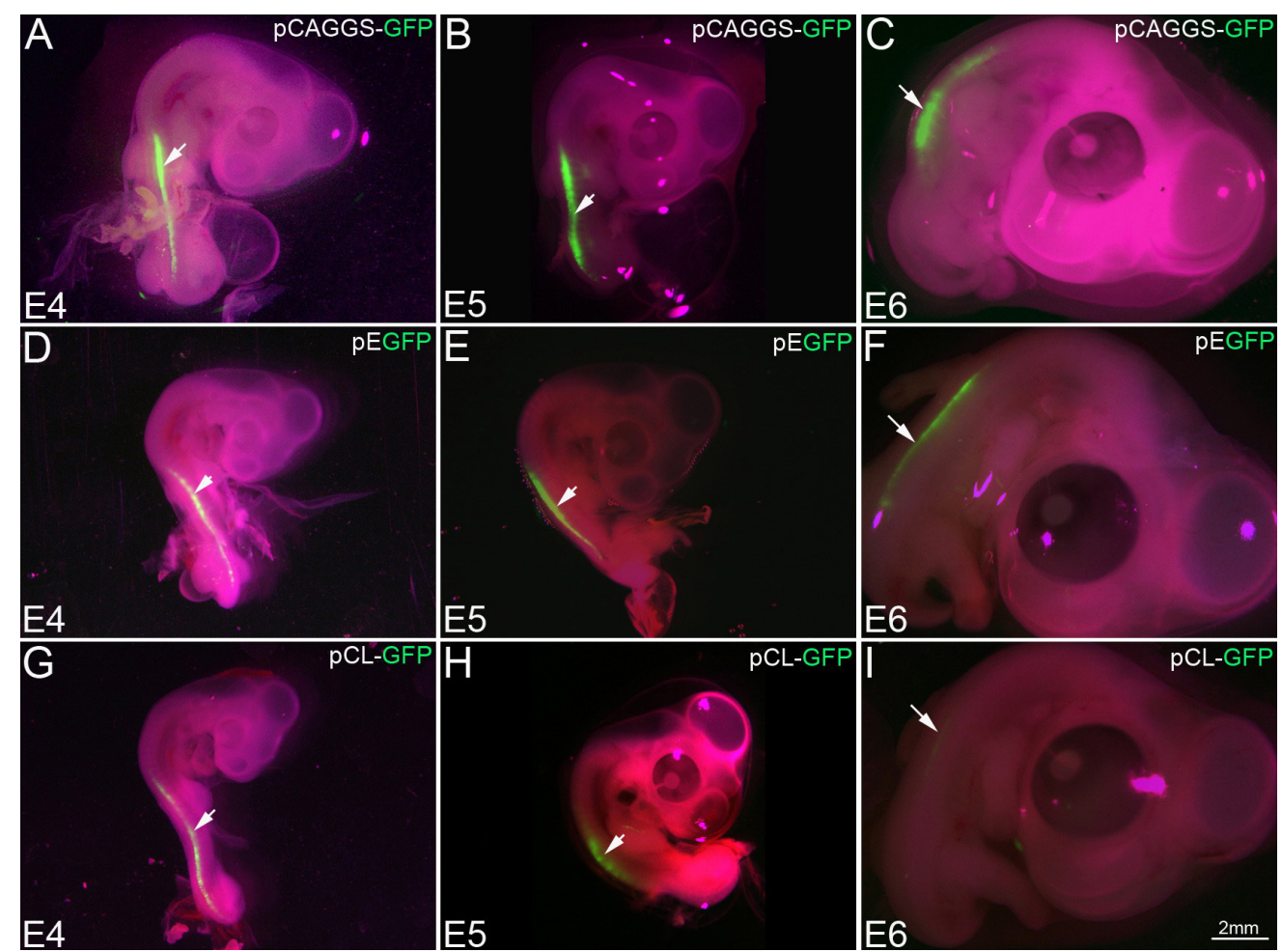

Figure 2. GFP expression for the three plasmids investigated at different stages after in ovo electroporation. A-C. GFP expression intensity of pCAGGS-GFP plasmid in the spinal cord at stage 24 (E4; A), stage 27 (E5; B) and stage 29 (E6; C). D-F. GFP expression intensity of pEGFP plasmid in the spinal cord at stage 24 (E4; D), stage 27 (E5; E) and stage 29 (E6; F). G-I. GFP expression intensity of pCL-GFP plasmid in the spinal cord at stage 24 (E4; G), stage 27 (E5; H) and stage 29 (E6; I). Scale bar $=2 \mathrm{~mm}$ in I applied for all panels.

\section{Slice analysis of GFP expression for the 3 plasmids in the chick embryonic spinal cord}

To compare the difference in intensity of the GFP expression level of the 3 investigated plasmids more clearly, the positive samples were sectioned, and the nuclei were stained by DAPI. At least 15 sections from each group were analyzed (obtained from at least 3 embryos 
from each group). The sections were collected at random from the regions along the anteriorposterior axis. Images from fluorescence microscopy were captured using the same exposure time. Then, the difference in GFP expression level for the 3 plasmids was analyzed at the same stage (E4, Figure 3A, B, C; E5, Figure 3E, F, G; E6, and Figure 3I, J, K). The expression intensity of GFP was compared at different stages for the same plasmid (pCAGGS-GFP, Figure 3A, E, I; pEGFP, Figure 3B, F, J; pCL-GFP, and Figure 3C, G, K). The arrow in Figure 3 shows (Figure 3A, B, C; 3E, F, G; and 3I, J, K) that pCAGGS-GFP had the strongest expression intensity of the 3 plasmids at each stage. The bar charts show that the intensity of GFP expression of the 3 plasmids differed (E4: Figure 3D; E5: Figure 3H; E6: Figure 3L). GFP fluorescence from the pCL-GFP-positive samples was significantly lower at electroporation after $72 \mathrm{~h}$ (Figure 3L). No significant difference was obtained for the normalized controls (data not shown).
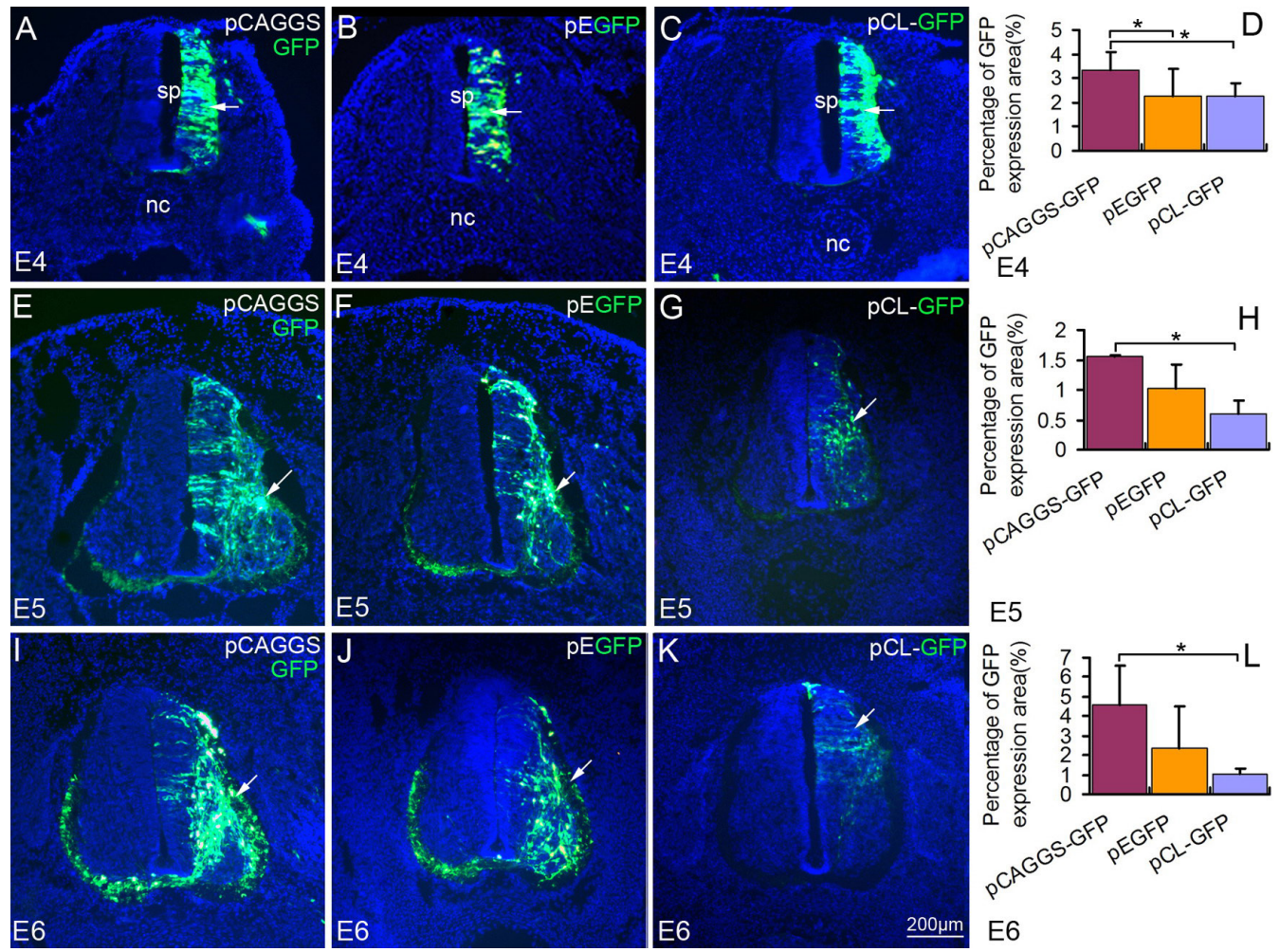

Figure 3. Slice analysis of GFP expression intensity for the three plasmids with different promoters in the chicken developing spinal cord. A. E. I. GFP expression intensity on slice of pCAGGS-GFP plasmid in the spinal cord at stage 24 (E4; A), stage 27 (E5; E) and stage 29 (E6; I). B. F. J. GFP expression intensity on slice of pEGFP plasmid in the spinal cord at stage 24 (E4; B), stage 27 (E5; F) and stage 29 (E6; J). C. G. K. GFP expression intensity on slice of pCL-GFP plasmid in the spinal cord at stage 24 (E4; C), stage 27 (E5; G) and stage 29 (E6; K). D. H. L. Percentage of GFP expression area occupied and captured at stage 24 (E4; D), stage 27 (E5; H) and stage 29 (E6; L) $(* \mathrm{P}<0.05$; each group selected 3 embryos and each embryo selected 5 sections at least). Scale bar $=200 \mu \mathrm{m}$ in $\mathrm{K}$ for A-C, E-G and I-K. 


\section{DISCUSSION}

Chicken embryos are widely used in the fields of developmental biology and neurobiology. The chicken embryo also serves as a type of optimal model to analyze gene expression and function by in vivo electroporation. Plasmids may be injected into the spinal cord or tectum of the chicken central nervous system by a microinjection needle used for electroporation. Thus, plasmids containing target genes may enter cells in the living system. However, the intensity of plasmid expression depends on both electrical transfection parameters and on the promoter carried by the plasmid itself. Different promoter and enhancer elements decide the intensity of target gene expression in different species. In the present study, 3 plasmids carrying the GFP reporter gene were used to analyze the driving efficiency of different promoters. Here, we compared GFP expression intensity driven by certain promoters at different stages and by different promoters of the 3 plasmids at the same stage. Our results indicate that the driving efficiency of promoters in the 3 plasmids is different. While other factors must also affect the intensity of GFP expression, such as enhancers and the plasmid skeleton, promoters play a major role.

In vivo electroporation represents a rapid and efficient method for the transformation of exogenous DNA into the spinal cord or other tissues. This method allows us to conduct gain of function experiments for target genes of interest, or to perform loss of function studies by using specific shRNA constructs for target genes of interest. Furthermore, multiple DNA plasmids may be simultaneously electroporated, allowing us multiple gene function to be analyzed (de Melo and Blackshaw, 2011). Appropriate promoters are required to drive the transcription of target genes or specific shRNA.

The in vivo experiments of the chicken embryo conducted here show that the transcriptional activity of the AG promoter is higher compared to the CMV promoter, while the CMV promoter is higher compared to the SV40 promoter. From the sequence analysis of the 3 plasmids investigated, pCAGGS-GFP contained an AG promoter and an hCMV IE enhancer, which are necessary for GFP and target gene expression. In comparison, pEGFP contains a CMV IE promoter, which controls target gene and GFP expression. Finally, pCL-GFP contains 2 promoters, $\mathrm{AG}$ and $\mathrm{SV} 40$, in which the $\mathrm{AG}$ promoter drives target gene expression, and the SV40 promoter drives GFP expression. Based on our results, when the strong target gene expression is required in the chicken system, a plasmid containing the AG promoter, such as pCAGGS, should be selected. In some experiments, the target gene may only require moderate expression; hence, the plasmid containing the CMV promoter would be a good choice in such cases. In certain experiments, only weak target gene expression might be required, in which case the SV40 promoter would be useful.

In general, the target gene is expressed together with the reporter gene (for example, GFP) as a fusion protein; however, in most cases, GFP might affect the normal folding of target proteins. To solve this problem, one IRES sequence may be inserted between the target gene and the GFP under the same promoter. Of course, ideally, 2 different and separated promoters in 1 plasmid should be used to drive the expression of the target gene and GFP. Interestingly, pCL-GFP contains double promoters, which may be separately used for the overexpression of the target gene and reporter gene. There are a number of other plasmids with different promoters that were not investigated in the current study. In conclusion, this study provides experimental evidence confirming the importance of selecting appropriate promoters for the correct expression of exogenous genes in the chicken system. 


\section{ACKNOWLEDGMENTS}

We thank Dr. Marcelle for providing the plasmid pCL-GFP and Ms. Fan for providing experimental assistance. Research supported by a grant from the National Science Foundation of China (\#31000475).

\section{REFERENCES}

Chang Y, Li Y, Hu J, Guo J, et al. (2013). Adenovirus vector-mediated expression of TMEM166 inhibits human cancer cell growth by autophagy and apoptosis in vitro and in vivo. Cancer Lett. 328: 126-134.

Chesnutt C and Niswander L (2004). Plasmid-based short-hairpin RNA interference in the chicken embryo. Genesis 39: 73-78.

Das RM, Van Hateren NJ, Howell GR, Farrell ER, et al. (2006). A robust system for RNA interference in the chicken using a modified microRNA operon. Dev. Biol. 294: 554-563.

de Melo J and Blackshaw S (2011). In vivo electroporation of developing mouse retina. J. Vis. Exp. Doi: 10.3791/2847.

Hamburger V and Hamilton HL (1992). A series of normal stages in the development of the chick embryo. Dev. Dyn. 195: 231-272.

Hong S, Hwang DY, Yoon S, Isacson O, et al. (2007). Functional analysis of various promoters in lentiviral vectors at different stages of in vitro differentiation of mouse embryonic stem cells. Mol. Ther. 15: 1630-1639.

Katahira T and Nakamura H (2003). Gene silencing in chick embryos with a vector-based small interfering RNA system. Dev. Growth Differ. 45: 361-367.

Lin J, Lemke C, Redies C, Yan X, et al. (2011). ADAM17 overexpression promotes angiogenesis by increasing blood vessel sprouting and pericyte number during brain microvessel development. Int. J. Dev. Biol. 55: 961-968.

Luo J and Redies C (2005). Ex ovo electroporation for gene transfer into older chicken embryos. Dev. Dyn. 233: $1470-1477$.

Muramatsu T, Mizutani Y, Ohmori Y and Okumura J (1997). Comparison of three nonviral transfection methods for foreign gene expression in early chicken embryos in ovo. Biochem. Biophys. Res. Commun. 230: 376-380.

Nakamura H, Katahira T, Sato T, Watanabe Y, et al. (2004). Gain- and loss-of-function in chick embryos by electroporation. Mech. Dev. 121: 1137-1143.

Rhodius VA, Mutalik VK and Gross CA (2012). Predicting the strength of UP-elements and full-length E. coli $\sigma \mathrm{E}$ promoters. Nucleic Acids Res. 40: 2907-2924.

Sauka-Spengler T and Barembaum M (2008). Gain- and loss-of-function approaches in the chick embryo. Methods Cell Biol. 87: 237-256.

Stern CD (2005). The chick; a great model system becomes even greater. Dev. Cell 8: 9-17.

Voiculescu O, Papanayotou C and Stern CD (2008). Spatially and temporally controlled electroporation of early chick embryos. Nat. Protoc. 3: 419-426. 